Editorial

\title{
Widding the Use of Insulin Sensitizers to Patients with Polycystic Ovarian Syndrome-A Late, but Wise Decision
}

\section{Ampliando o uso de sensibilizadores de insulina às pacientes com síndrome do ovário policístico - Atrasada, mas uma sábia decisão}

\author{
Marcos Felipe Silva de Sá1우 \\ ${ }^{1}$ Department of Gynecology and Obstetrics, Faculty of Medicine, \\ Universidade de São Paulo, Ribeirão Preto, SP, Brazil
}

Rev Bras Ginecol Obstet 2019;41:137-141.

Polycystic Ovarian Syndrome (PCOS) is the leading endocrine disorder in women of childbearing age with a prevalence of more than $15 \%$ among this group. ${ }^{1}$ These women are predisposed to obesity, have a predominance of the abdominal obesity phenotype associated with the hyperandrogenic state, insulin resistance (IR) and compensatory hyperinsulinemia. ${ }^{2,3}$ It is estimated that $80 \%$ of women with PCOS have IR, and this scenario worsens in the presence of obesity. ${ }^{4,5}$

A few authors suggest that there are abnormalities in the energy expenditure of women with PCOS, resulting from a reduction in the resting metabolic rate, especially in those with IR. ${ }^{6}$ In PCOS, has been considered a lower postprandial response of gastrointestinal hormones involved in neural control of food intake. ${ }^{7-10}$ Food intake is controlled by complex interrelationships between homeostatic mechanisms that regulate caloric intake through a neuroendocrine system involving central and peripheral signals as well as mechanisms related to eating behavior. The central mechanisms are regulated by learning, memory and the reward system that acts in the mesolimbic circuit present in the central nervous system. ${ }^{11,12}$ Gastrointestinal peripheral signs encompass several hormones that act on hunger (ghrelin) and satiation/satiety (cholecystokinin, YY peptide, oxintomodulin, glucagon like peptide - GLP-1, glucagon and amylin), and play an important role in regulating appetite and caloric intake.

Some studies have shown that the plasma levels of GLP-1 and PYY in the postprandial period are lower in women with PCOS, ${ }^{13,14}$ and the exogenous administration of these hormones triggers the sensation of fullness and reduces caloric intake in animals and humans. ${ }^{15}$ Agonists of the GLP-1 have been used to promote weight loss in diabetic patients, and

(D) Marcos Felipe Silva de Sá's ORCID is https://orcid.org/0000-00024813-6404.

Address for correspondence Marcos Felipe Silva de Sá, PhD, Av. Bandeirantes, 3900, 14049-900, Vila Monte Alegre, Ribeirão Preto,

SP, Brazil

(e-mail: marcosfelipe@fmrp.usp.br). there is suggestion that these drugs could also benefit patients with PCOS. ${ }^{16-19}$

Signs of body fat storage, such as insulin, leptin and adiponectin, also participate in the process of controlling food intake, as they indicate the state of energy reserve and alter appetite when necessary. ${ }^{20}$ Ghrelin is a gastrointestinal hormone known to stimulate food intake and its secretion is regulated by insulin levels and body reserves. ${ }^{21,22}$ Women with PCOS have lower levels of fasting ghrelin and less postprandial suppression of ghrelin compared with obese women without PCOS. ${ }^{7,9}$ A common finding in the literature is the negative correlation between insulin resistance and ghrelin plasma concentrations. ${ }^{23-25}$ When studying two groups of obese women (PCOS versus non-PCOS), we observed a lower ratio of preprandial ghrelin/insulin in the PCOS group. The postprandial ghrelin response was similar in both groups, and there was a significant negative correlation between ghrelin and insulin in both groups as well. However, when the patients were split into two groups, according to IR and regardless of PCOS, the negative correlation between ghrelin and insulin did not occur in the group with IR. These results suggest that the mechanism of ghrelin suppression by insulin may be impaired in women with IR, regardless of PCOS. ${ }^{25}$ Since IR is highly prevalent in obese PCOS patients, with rates above $90 \%,{ }^{4}$ control of the satiation/satiety process is likely to function inadequately in these patients. In fact, in the patients we studied, an earlier increase in postprandial hunger was observed in obese women with PCOS than in obese women without PCOS. These observations have already been described by others, ${ }^{7}$ indicating the possibility that patients with PCOS would have impaired regulatory mechanisms of ingestion and satiation/satiety when compared with women without PCOS, which could explain the greater difficulty in weight loss of women with PCOS.

Copyright $\odot 2019$ by Thieme Publicações License terms Ltda, Rio de Janeiro, Brazil

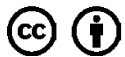


Weight loss has been the main option for treating PCOS. Low-glycemic diets and lifestyle changes associated or not to physical exercise lead to weight loss, reduce hyperandrogenism, increase ovulation and pregnancy rates, and are also beneficial in reducing hyperinsulinemia and its metabolic consequences. ${ }^{26-30}$ The main limitation of this type of therapy are the difficulties of maintaining weight loss for an extended period of time, and relapses are unfortunately frequent. Consequently, there is a growing use of bariatric surgery for the treatment of obesity in women with PCOS, which has significantly better results in terms of sustainable weight loss. ${ }^{31}$

The association of PCOS with IR, and, consequently, with hyperinsulinemia is known for decades, and in addition to its effect on the reproductive system, it is related to the high prevalence of obesity, glucose intolerance (GI), type 2 diabetes mellitus (DM2), dyslipidemia and vascular inflammatory processes. Consistent pictures with the metabolic syndrome, elevated triglycerides and LDL, and reduced HDL are frequent. ${ }^{32,33}$

By considering these evidences, it is reasonable to propose that the main focus addressed in therapeutic strategies for weight loss in PCOS should also take into account the approach to IR. The use of insulin sensitizers reduces endogenous insulin requirements, with preservation of $\beta$ cells, and it may protect insulin-resistant patients from developing DM2. ${ }^{34,35}$

Despite the extensive literature on the deleterious role of IR in the evolution of PCOS and its association with changes in control mechanisms of caloric intake and satiation/satiety and the benefits of insulin sensitizers in disease therapy, important international societies have been reluctant to recommend its use associated with diet, lifestyle and physical exercise as the primary therapy of PCOS.

In the first consensus published as a group in 2004, even though the European Society of Human Reproduction (ESHERE) and the American Society of Reproductive Medicine (ASRM) recognized the high prevalence of IR in women with PCOS, they did not recommend IR screening in these patients by considering the restricted validity of clinical tests for its diagnosis. The group recognized some studies that showed the progression of GI to DM2 may be delayed by lifestyle changes and pharmacological intervention with insulin sensitizers, but did not recommend the use of these drugs routinely in the treatment of PCOS with IR. ${ }^{1}$

Four years later, the Thessaloniki ESHRE/ASRM-Sponsored PCOS Consensus Workshop Group proposed that "before any intervention is initiated, preconceptional counseling should be emphasized on the importance of lifestyle, especially weight reduction and exercise in overweight women, smoking, and alcohol consumption," and recommended restricting the use of metformin (MTF) to women with PCOS and GI. Thus, the presence of IR should not be a reason for prescribing MTF. $^{36}$

If IR is a step preceding GI, it did not seem reasonable to wait for the worsening of the metabolic profile in order to introduce insulin-sensitizing therapy. Considering the poor results obtained with lifestyle and diet changes on weight loss, we believe it is unreasonable to let the patient stay for a longer time under high levels of insulinemia and all its consequences.

However, the ESHERE and ASRM maintained the same position in a new version of the Amsterdam consensus published in 2011: "Diet and lifestyle are the first choice in improving fertility and prevention of diabetes (Level B). Metformin may be used for impaired glucose tolerance (IGT) and DM2 (Level A.)." Management of women at risk for DM2 should include diet and lifestyle improvement as first-line treatment. Treatment with MTF is indicated to those patients with IGT who do not respond adequately to calorie restriction and lifestyle changes." ${ }^{37}$ The authors considered there is insufficient scientific evidence to recommend the use of insulin sensitizers beyond that situation, which indicates great caution in its use.

In a debate (pro $\mathrm{x}$ con) between two experts (Marshall and Dunaif), ${ }^{38}$ numerous justifications were offered for using MTF as an important adjuvant factor in the therapy of patients with PCOS, given the high prevalence of IR, obesity, GI, DM2, dyslipidemia, increased evidence of inflammatory process and metabolic syndrome. ${ }^{38}$ Marshal concluded that "the majority of evidence in adult women indicate that treatment of insulin resistance, either by lifestyle changes or metformin, leads to improvement in reproductive and metabolic abnormalities and probably reduces future development of diabetes and arterial disease." ${ }^{38}$ Although Dunaif does not support the use of insulin sensitizers in all women with PCOS, she acknowledges this measure could be taken in view of the difficulties of maintaining diet and lifestyle in a younger population. ${ }^{38}$ In fact, several other societies have recommended the use of MTF in youngsters and adolescents with PCOS as a first-line therapy, combined or not with oral contraceptives and antiandrogens for the treatment of hyperandrogenemia and its symptoms, reestablishment of menses, aid with reduction of weight and IR, aiming at the prevention of long-term cardiovascular complications, even in lean adolescents. ${ }^{39-43}$ The fight against hyperandrogenism plays a key role in the process of containing the metabolic degeneration of these patients. The risk of GI and/or DM2 is greater in women who have oligo-anovulation and hyperandrogenism and this risk is even greater if they are obese. $^{44}$

If the use of MTF has been practically a consensus for treating young, lean or obese, adolescents for some time, why not also apply it to adult women in the age group of 20 to 35 years, which corresponds to the prevailing age of patients with PCOS seeking care for the treatment of menstrual disorders and infertility? These are relatively young patients who may have their prognosis worsened and increased risk of metabolic syndrome if there is no intervention. Hence, it is reasonable to think that the benefits of insulins sensitizers could also reach this age group.

According to some authors, there are no data supporting the treatment of PCOS with MTF based on the measures of IR by arguing that the parameters for its calculation are not sensitive neither specific. ${ }^{38}$ However, other authors have 
contested this suggestion through the composition of various methods of calculations based on measurements of glucose and fasting insulin. ${ }^{4}$

Another argument against the use of MTF as first-line treatment for PCOS is the lack of data on the results of IR treatment per se in PCOS. Therefore, the metabolic assessment of women with PCOS should focus on detecting conditions that justify the intervention, such as GI, metabolic syndrome and elevated HDL levels. ${ }^{38}$ Metabolic syndrome and its indiviual components are common in patients with PCOS, particularly in those with elevated insulin levels (therefore, with IR) and increased weight. However, in patients with PCOS and normal body mass index (BMI), the diagnosis of metabolic syndrome is rare, although the literature shows that $19 \%$ of patients without metabolic syndrome may have $\mathrm{GI}^{32}$ and, certainly, a much higher percentage may have IR, since it can be found in up to $70 \%$ of non-obese patients with PCOS. ${ }^{4}$ For example, is there any doubt about IR in patients with Acanthosis Nigricans, even if they are lean?

In fact, randomized clinical trials in women with PCOS are needed to check the efficacy of insulin sensitizers for the improvement of metabolic endpoints. The criticism of the literature is that much information was obtained in studies investigating non-metabolic endpoints in PCOS or that nonPCOS populations were studied. ${ }^{36-38}$ Randomized clinical trials are an essential tool in the construction of scientific evidence for clinical practice, although not always the most useful for some evaluations. Generally, their performance is complex, costly, with numerous operational difficulties, and many of them do not have adequate sample size for evaluating therapeutic practices. Moreover, although the findings of controlled therapeutic trials are statistically significant, there is no guarantee they will serve the totality of individuals. Considering the vast medical scenario, there are few situations in which decision-making is supported by evidence-based medicine. Most health practices are based on experimental cohort or case-control studies, or even non controlled observation of a set of cases.

In the specific case of PCOS, it is easy to imagine how difficult it would be to carry out a randomized clinical study to identify patients at risk and conduct a longitudinal study of cohorts of women with PCOS beyond 60 years of age to determine with greater precision the time and efficacy of the interventions studied for answering if insulin sensitizers used in patients with IR definitely prevent GI or DM2. Many patients would be followed up for decades and after entering their postmenopausal period.

The lack of these studies may mean we do not have definitive answers to elucidate endpoints. However, the lack of these answers should not be enough reason to stop prescribing those drugs for these patients. Numerous evidences based on experimental cohort or epidemiological studies also have their value. By exclusively valuing evidence-based medicine obtained in randomized clinical trials or systematic reviews with meta-analysis, we certainly fail to cover numerous clinical situations, as in the case of PCOS. In these situations, it is absolutely pertinent to use evidence based on medicine rather than evidence-based medicine for decision-making in the face of individualized clinical situations. Thus, studies with less rigorous designs may have their place in everyday practice for the care of a particular individual. $^{45}$

Although the FDA has approved MTF for DM2 treatment, it has been used off label for more than 40 years to reduce the signs and symptoms of PCOS and normalize the various parameters that assess risks for DM2, obese and non-obese, children, adolescents and adults. ${ }^{35,41,46-49}$ For these reasons, we have been asking ourselves: why not routinely prescribe it for hyperandrogenic and obese patients with PCOS, regardless of whether they have GI or DM2? Should we expect the worsening of the insulin resistance picture for its prescription $?^{50}$

Finally, in the last ASRM/ESHERE consensus, it was recognized the validity of a more comprehensive use of MTF in the treatment of PCOS, based on evidence of its clear benefits in the specific PCOS group or subgroups by improving weight, BMI, hip-waist ratio, testosterone and glucose, especially in obese patients, since metabolic benefits are more accentuated in patients with increased BMI. Thus, in addition to lifestyle changes, the use of MTF was recommended in adult women with PCOS for the treatment of overweight and hormonal and metabolic changes. The benefit of MTF use in adolescents diagnosed with PCOS was also considered. ${ }^{51}$ Although not specified, note that a more comprehensive introduction of insulin sensitizers may also contribute to a better observation and comprehension of the effects of IR on the control mechanisms of ingestion/satiation/satiety that are possibly committed in PCOS.

Faced with so much evidence, restricting the use of insulin sensitizers for patients with PCOS no longer made sense. This new position of the most important societies of the specialty expands the range to patients with PCOS that can benefit from these drugs. The restriction on its use until recently may have deprived thousands of patients of the benefit of reducing IR and its consequences. It is known that IR is related to endothelial dysfunction and that it progresses with time. Endothelial dysfunction caused by IR has been related to reduced nitric oxide bioavailability and changes in endothelial regeneration. Research on the use of insulin sensitizers for the treatment of polycystic ovaries in rats has shown that endothelial dysfunction is the direct result of hyperandrogenism induced by IR, and treatment with MTF improves insulin sensitivity and blood pressure and reestablishes normal endothelial function, even with the weight gain of animals. ${ }^{52}$ Some studies have also shown that pioglitazone, an insulin sensitizer, has effects on glucose homeostasis, and exerts pleiotropic effects, thereby improving endothelial dysfunction. ${ }^{53}$

Professionals from all over the world follow guidelines of the major American and European specialty societies, which impact direct on the therapeutics adopted globally. Therefore, the wider use of insulin sensitizers brings great prospects of more concrete clinical results regarding this therapy for both young and adult patients with PCOS, especially obese patients, as a first-line treatment. We believe 
that gynecologists are able to prescribe this drug because international experience has shown this is a low-risk product that has been increasingly used in patients with PCOS, youngsters, adolescents and adults, obese or non-obese, including pregnant patients with PCOS and gestational diabetes, and in hypertension during pregnancy. ${ }^{54}$

\section{Conflicts of Interest \\ The Author Has No Conflicts Of Interest To Declare.}

\section{References}

1 Rotterdam ESHRE/ASRM-Sponsored PCOS Consensus Workshop Group. Revised 2003 consensus on diagnostic criteria and longterm health risks related to polycystic ovary syndrome. Fertil Steril 2004;81:19-25. Doi: 10.1016/j.fertnstert.2003.10.004

2 Gambineri A, Pelusi C, Vicennati V, Pagotto U, Pasquali R. Obesity and the polycystic ovary syndrome. Int J Obes Relat Metab Disord 2002;26(07):883-896. Doi: 10.1038/sj.ijo.0801994

3 Penaforte FRO, Japur CC, Diez-Garcia RW, Chiarello PG. Upper trunk fat assessment and its relationship with metabolic and biochemical variables and body fat in polycystic ovary syndrome. J Hum Nutr Diet 2011;24(01):39-46. Doi: 10.1111/j.1365-277X.2010.01130.x

4 Carmina E, Lobo RA. Use of fasting blood to assess the prevalence of insulin resistance in women with polycystic ovary syndrome. Fertil Steril 2004;82(03):661-665. Doi: 10.1016/j.fertnstert.2004.01.041

5 Hoeger KM, Oberfield SE. Do women with PCOS have a unique predisposition to obesity? Fertil Steril 2012;97(01):13-17. Doi: 10.1016/j.fertnstert.2011.11.026

6 Georgopoulos NA, Saltamavros AD, Vervita V, et al. Basal metabolic rate is decreased in women with polycystic ovary syndrome and biochemical hyperandrogenemia and is associated with insulin resistance. Fertil Steril 2009;92(01):250-255. Doi: 10.1016/j.fertnstert.2008.04.067

7 Moran LJ, Noakes M, Clifton PM, et al. Ghrelin and measures of satiety are altered in polycystic ovary syndrome but not differentially affected by diet composition. J Clin Endocrinol Metab 2004;89(07):3337-3344. Doi: 10.1210/jc.2003-031583

8 Hirschberg AL, Naessén S, Stridsberg M, Byström B, Holtet J. Impaired cholecystokinin secretion and disturbed appetite regulation in women with polycystic ovary syndrome. Gynecol Endocrinol 2004;19(02):79-87. Doi: 10.1080/09513590400002300

9 Moran LJ, Noakes M, Clifton PM, et al. Postprandial ghrelin, cholecystokinin, peptide YY, and appetite before and after weight loss in overweight women with and without polycystic ovary syndrome. Am J Clin Nutr 2007;86(06):1603-1610. Doi: 10.1093/ ajen/86.5.1603

10 Barber TM, Casanueva FF, Karpe F, et al. Ghrelin levels are suppressed and show a blunted response to oral glucose in women with polycystic ovary syndrome. Eur J Endocrinol 2008; 158(04):511-516. Doi: 10.1530/EJE-07-0683

11 Harrold JA, Dovey TM, Blundell JE, Halford JC. CNS regulation of appetite. Neuropharmacology 2012;63(01):3-17. Doi: 10.1016/j. neuropharm.2012.01.007

12 Kanoski SE. Cognitive and neuronal systems underlying obesity. Physiol Behav 2012;106(03):337-344. Doi: 10.1016/j.physbeh. 2012.01.007

13 Zwirska-Korczala K, Sodowski K, Konturek SJ, et al. Postprandial response of ghrelin and PYY and indices of low-grade chronic inflammation in lean young women with polycystic ovary syndrome. J Physiol Pharmacol 2008;59(Suppl 2):161-178

$14 \mathrm{Lin} \mathrm{T,} \mathrm{Li} \mathrm{S,} \mathrm{Xu} \mathrm{H,} \mathrm{et} \mathrm{al.} \mathrm{Gastrointestinal} \mathrm{hormone} \mathrm{secretion} \mathrm{in} \mathrm{women}$ with polycystic ovary syndrome: an observational study. Hum Reprod 2015;30(11):2639-2644. Doi: 10.1093/humrep/dev231
15 Neary NM, Small CJ, Druce MR, et al. Peptide YY3-36 and glucagon-like peptide-17-36 inhibit food intake additively. Endocrinology 2005;146(12):5120-5127. Doi: 10.1210/en.2005-0237

16 Elkind-Hirsch K, Marrioneaux O, Bhushan M, Vernor D, Bhushan R. Comparison of single and combined treatment with exenatide and metformin on menstrual cyclicity in overweight women with polycystic ovary syndrome. J Clin Endocrinol Metab 2008;93(07): 2670-2678. Doi: 10.1210/jc.2008-0115

17 Tanaka K, Saisho Y, Kawai T, et al. Efficacy and safety of liraglutide monotherapy compared with metformin in Japanese overweight/ obese patients with type 2 diabetes. Endocr J 2015;62(05): 399-409. Doi: 10.1507/endocrj.EJ14-0602

18 Liu X, Zhang Y, Zheng SY, et al. Efficacy of exenatide on weight loss, metabolic parameters and pregnancy in overweight/obese polycystic ovary syndrome. Clin Endocrinol (Oxf) 2017;87(06): 767-774. Doi: 10.1111/cen.13454

19 Tsilchorozidou T, Batterham RL, Conway GS. Metformin increases fasting plasma peptide tyrosine tyrosine (PYY) in women with polycystic ovarian syndrome (PCOS). Clin Endocrinol (Oxf) 2008; 69(06):936-942. Doi: 10.1111/j.1365-2265.2008.03285.x

20 Wynne K, Stanley S, McGowan B, Bloom S. Appetite control J Endocrinol 2005;184(02):291-318. Doi: 10.1677/joe.1.05866

21 Cummings DE, Purnell JQ Frayo RS, Schmidova K, Wisse BE, Weigle DS. A preprandial rise in plasma ghrelin levels suggests a role in meal initiation in humans. Diabetes 2001;50(08): 1714-1719. Doi: 10.2337/diabetes.50.8.1714

22 Micic D, Sumarac-Dumanovic M, Kendereski A, et al. Total ghrelin levels during acute insulin infusion in patients with polycystic ovary syndrome. J Endocrinol Invest 2007;30(10):820-827. Doi: $10.1007 / \mathrm{BF} 03349222$

23 Schöfl C, Horn R, Schill T, Schlösser HW, Müller MJ, Brabant G. Circulating ghrelin levels in patients with polycystic ovary syndrome. J Clin Endocrinol Metab 2002;87(10):4607-4610. Doi: 10.1210/jc.2002-020505

24 Waśko R, Komarowska H, Warenik-Szymankiewicz A, Sowiński J. Elevated ghrelin plasma levels in patients with polycystic ovary syndrome. Horm Metab Res 2004;36(03):170-173. Doi: 10.1055/ s-2004-814341

25 Japur CC, Diez-Garcia RW, de Oliveira Penaforte FR, de Sá MFS Imbalance between postprandial ghrelin and insulin responses to an ad libitum meal in obese women with polycystic ovary syndrome. Reprod Sci 2014;21(08):1020-1026. Doi: 10.1177/ 1933719114522521

26 Wright CE, Zborowski JV, Talbott EO, McHugh-Pemu K, Youk A. Dietary intake, physical activity, and obesity in women with polycystic ovary syndrome. Int J Obes Relat Metab Disord 2004; 28(08):1026-1032. Doi: 10.1038/sj.ijo.0802661

27 Reaven GM. The insulin resistance syndrome: definition and dietary approaches to treatment. Annu Rev Nutr 2005; 25:391-406. Doi: 10.1146/annurev.nutr.24.012003.132155

28 Bruner B, Chad K, Chizen D. Effects of exercise and nutritional counseling in women with polycystic ovary syndrome. Appl Physiol Nutr Metab 2006;31(04):384-391. Doi: 10.1139/h06-007

29 Norman RJ, Davies MJ, Lord J, Moran LJ. The role of lifestyle modification in polycystic ovary syndrome. Trends Endocrinol Metab 2002;13(06):251-257. Doi: 10.1016/S1043-2760(02) 00612-4

30 Hoeger KM. Exercise therapy in polycystic ovary syndrome. Semin Reprod Med 2008;26(01):93-100. Doi: 10.1055/s-2007-992929

31 Escobar-Morreale HF, Botella-Carretero JI, Alvarez-Blasco F, Sancho J, San Millán JL. The polycystic ovary syndrome associated with morbid obesity may resolve after weight loss induced by bariatric surgery. J Clin Endocrinol Metab 2005;90(12):6364-6369. Doi: $10.1210 /$ jc.2005-1490

32 Dunaif A. Insulin resistance and the polycystic ovary syndrome: mechanism and implications for pathogenesis. Endocr Rev 1997; 18(06):774-800. Doi: 10.1210/edrv.18.6.0318 
33 Ehrmann DA, Liljenquist DR, Kasza K, Azziz R, Legro RS, Ghazzi MN; PCOS/Troglitazone Study Group. Prevalence and predictors of the metabolic syndrome in women with polycystic ovary syndrome.J Clin Endocrinol Metab 2006;91(01):48-53. Doi: 10.1210/jc.2005-1329

34 Buchanan TA, Xiang AH, Peters RK, et al. Preservation of pancreatic beta-cell function and prevention of type 2 diabetes by pharmacological treatment of insulin resistance in high-risk hispanic women. Diabetes 2002;51(09):2796-2803

35 Knowler WC, Barrett-Connor E, Fowler SE, et al; Diabetes Prevention Program Research Group. Reduction in the incidence of type 2 diabetes with lifestyle intervention or metformin. $\mathrm{N}$ Engl J Med 2002;346(06):393-403. Doi: 10.1056/NEJMoa012512

36 Thessaloniki ESHRE/ASRM-Sponsored PCOS Consensus Workshop Group. Consensus on infertility treatment related to polycystic ovary syndrome. Fertil Steril 2008;89:505-522. Doi: 10.1016/j.fertnstert.2007.09.041

37 Amsterdam ESHRE/ASRM-Sponsored 3rd PCOS Consensus Workshop Group. Consensus on women's health aspects of polycystic ovary syndrome (PCOS). Hum Reprod 2012;27:14-12. Doi: 10.1093/ humrep/der396

38 Marshall JC, Dunaif A. Should all women with PCOS be treated for insulin resistance? Fertil Steril 2012;97(01):18-22. Doi: 10.1016/ j.fertnstert.2011.11.036

39 Wild RA, Carmina E, Diamanti-Kandarakis E, et al. Assessment of cardiovascular risk and prevention of cardiovascular disease in women with the polycystic ovary syndrome: a consensus statement by the Androgen Excess and Polycystic Ovary Syndrome (AE-PCOS) Society. J Clin Endocrinol Metab 2010;95(05):2038-2049. Doi: 10.1210/jc.2009-2724

40 Goodman NF, Cobin RH, Futterweit W, Glueck JS, Legro RS, Carmina E; American Association of Clinical Endocrinologists (AACE); American College of Endocrinology (ACE); Androgen Excess and PCOS Society (AES). American Association of Clinical Endocrinologists, American College of Endocrinology, and Androgen Excess and PCOS Society Disease state clinical review: guide to the best practices in the evaluation and treatment of Polycystic Ovary Syndrome-part 1. Endocr Pract 2015;21(11):1291-1300. Doi: 10.4158/EP15748.DSC

41 Geller DH, Pacaud D, Gordon CM, Misra M; of the Drug and Therapeutics Committee of the Pediatric Endocrine Society. of the Drug and Therapeutics Committee of the Pediatric Endocrine Society. State of the art review: emerging therapies: the use of insulin sensitizers in the treatment of adolescents with polycystic ovary syndrome (PCOS). Int J Pediatr Endocrinol 2011;2011:9. Doi: 10.1186/1687-9856-2011-9

42 Silfen ME, Denburg MR, Manibo AM, et al. Early endocrine, metabolic, and sonographic characteristics of polycystic ovary syndrome (PCOS): comparison between nonobese and obese adolescents. J Clin Endocrinol Metab 2003;88(10):4682-4688. Doi: $10.1210 /$ jc.2003-030617
43 Hsia Y, Dawoud D, Sutcliffe AG, Viner RM, Kinra S, Wong IC. Unlicensed use of metformin in children and adolescents in the UK. Br J Clin Pharmacol 2012;73(01):135-139. Doi: 10.1111/ j.1365-2125.2011.04063.x

44 Barber TM, Wass JA, McCarthy MI, Franks S. Metabolic characteristics of women with polycystic ovaries and oligo-amenorrhoea but normal androgen levels: implications for the management of polycystic ovary syndrome. Clin Endocrinol (Oxf) 2007;66(04): 513-517. Doi: 10.1111/j.1365-2265.2007.02764.x

45 Horwitz RI, Hayes-Conroy A, Caricchio R, Singer BH. From evidence based medicine to medicine based evidence. Am J Med 2017;130(11):1246-1250. Doi: 10.1016/j.amjmed.2017.06.012

46 Mathur R, Alexander CJ, Yano J, Trivax B, Azziz R. Use of metformin in polycystic ovary syndrome. Am J Obstet Gynecol 2008;199(06): 596-609. Doi: 10.1016/j.ajog.2008.09.010

47 Hoeger K, Davidson K, Kochman L, Cherry T, Kopin L, Guzick DS. The impact of metformin, oral contraceptives, and lifestyle modification on polycystic ovary syndrome in obese adolescent women in two randomized, placebo-controlled clinical trials. J Clin Endocrinol Metab 2008;93(11):4299-4306. Doi: 10.1210/ jc.2008-0461

48 Legro RS. Obesity and PCOS: implications for diagnosis and treatment. Semin Reprod Med 2012;30(06):496-506. Doi: 10.1055/s-0032-1328878

49 Salpeter SR, Buckley NS, Kahn JA, Salpeter EE. Meta-analysis: metformin treatment in persons at risk for diabetes mellitus. Am J Med 2008;121(02):149-157.e2. Doi: 10.1016/j.amjmed.2007. 09.016

50 Soares Júnior JM, Sa MF, Baracat EC. [Should insulin resistance be always treated in Polycystic Ovary Syndrome?] Rev Bras Ginecol Obstet 2014;36(02):47-49. Doi: 10.1590/S0100-720320140002 00001

51 Teede HJ, Misso ML, Costello MF, et al; International PCOS Network. Recommendations from the international evidence-based guideline for the assessment and management of polycystic ovary syndrome. Fertil Steril 2018;110(03):364-379. Doi: 10.1016/j. fertnstert.2018.05.004

52 Hurliman A, Keller Brown J, Maille N, Mandala M, Casson P, Osol G. Hyperandrogenism and insulin resistance, not changes in body weight, mediate the development of endothelial dysfunction in a female rat model of Polycystic Ovary Syndrome (PCOS). Endocrinology 2015;156(11):4071-4080. Doi: 10.1210/en.2015-1159

53 Radenković M. Pioglitazone and endothelial dysfunction: pleiotropic effects and possible therapeutic implications. Sci Pharm 2014;82(04):709-721. Doi: 10.3797/scipharm.1407-16

54 Romero R, Erez O, Hüttemann M, et al. Metformin, the aspirin of the 21st century: its role in gestational diabetes mellitus, prevention of preeclampsia and cancer, and the promotion of longevity. Am J Obstet Gynecol 2017;217(03):282-302. Doi: 10.1016/ j.ajog.2017.06.003 